\title{
Nocebo phenomenon
}

\section{Samorindo Peci - Federica Peci}

Ce.ri.fo.s. Centro Ricerca Milano

doi: $10.7358 /$ neur-2016-020-peci

samorindo.peci@cerifos.it

\section{Abstract}

The term "Nocebo" (latin, "I shall harm") was coined in 1961 by Kennedy to highlight the negative counterpart of the placebo phenomenon so as to be able to distinguish the adverse from the beneficial effects of placebos. It concerns the occurrence of adverse effects because they are expected to develop, attributed to the intervention. Originally, in analogy with placebo, the term was used to describe an inactive substance or ineffective treatment with the aim to elicit negative expectations by verbally suggesting an increase in symptoms. Today, the nocebo effect is an ill effect caused by the suggestion or belief that something is harmful. It's considered a psychobiological phenomenon deriving from a negative psychosocial context surrounding the treatment, resulting in a worsening of symptoms caused by negative expectations and in without the administration of any inert substance. If compared to placebo effect, this phenomenon has only recently received wider attention from basic scientists and clinicians but is still poorly understood. The reason might be found in the stressful and anxiogenic nature of this procedure limiting its ethic al investigation.

Keywords: Nocebo; Medication; Clinical practice

\section{PSYCHOLOGICAL MECHANISMS}

Expectancy and classical conditioning are the main mechanisms for generating nocebo effects to have been extensively studied and to be well supported by behavioral data. Moreover, the patients' psychological characteristics and situational/contextual influences are fundamental factors for the occurrence of nocebo effect (Barsky, Saintfort, Rogers \& Borus, 2002).

Negative expectations can lead to a worsening of health conditions or the nocebo effect (Moerman, 1981). Information about negative outcomes, 
provided by physicians to trial subjects or gathered from sources including other patients and the internet make patients more likely to develop them. These information shall generate the very side-effect resembling the information given. A placebo-controlled trial of aspirin for unstable angina, led by Myers and Cairns in 1987, illustrates the role of these negative expectations. Researchers at three medical centers undertook the study of aspirin in heart patients and came up with an unexpected result. At two locations, patients were warned of possible gastrointestinal problems, one of the most common side effects of repeated use of aspirin. At the other location, patients received no such warning. Those warned about the gastrointestinal problems were almost three times as likely to experience the side effect. Though the evidence of actual stomach damage such as ulcers was the same for all three groups, those with the most information about the prospect of minor problems were the most likely to experience the pain.

The response to a drug or a treatment may be modified by the information given to the patient about it. In 1999, Flaten, Simonsen and Olsen demonstrated that by simply administrating a muscle relaxant to a group of patients. Among them, those who were told it was a stimulant reported greater muscle tension than those who were told it was a relaxant.

Expectations may also induce symptoms in healthy individuals. An emblematic study was done by Schweiger and Parducci in 1981. 34 college students were told an electric current would be passed through their heads, and the researchers warned that the experience could cause a headache. Though not a single volt of current was used, more than two-thirds of the students reported headaches. Bingel et al. (2011) pointed out that an individual's expectation of a drug's effect critically influences its therapeutic efficacy. They exposed healthy volunteers to experimental pain stimuli and gave them an active pain-reducing medication and told them that the medication would intensify their pain once the infusion of medication would cease. Interestingly, the participants experienced a pain increase to the extent that the effect of the active treatment was nullified.

If patients have experienced side effects to drugs in the past, they are likely to manifest side effects to a prescribed medication, in spite of its specific pharmacological actions. This is a result of prior conditioning. For example, among chemotherapy patients, conditioned nausea occurs in as many as $29 \%$ of them (Morrow, Roscoe, Kirshner, Hynes \& Rosenbluth, 1998). They become deeply nauseated even when they encounter a stimulus that can be associated with chemotherapy. Conditioned responses can be observed also in healthy non-patients. Accordingly to Lees-Haley and Brown (1992), when a social unit, like a family, a group of workers or an entire community think erroneously they have been exposed to a toxic substance, 
the members of that group experience an increased incidence of distressing somatic symptoms that they ascribe to the supposed exposure.

Although a prior negative patient experience with physicians constitutes the commonest cause for a difficult patient encounter, specific psychological disorders also contribute, including somatoform disorders, personality disorders, and adjustment disorders, with associated feelings of guilt and/or obsession. Anxious and depressed patients react worse to the treatment than a person in a better mood and psychically labile persons feel the side effects of the medications more often, because they have the tendency toward somatization, symptom amplification and a heightened awareness of bodily sensation. Furthermore, traits such as neuroticism, pessimism and type A personalities may predispose individuals to the nocebo effect phenomenon.

The context and the environment in which the medication is given influence the side effect reporting (Barsky, 1983). This mechanism for generating nocebo can be noted in everyday clinical practice, but there are little empirical studies about it. The importance of the context is shown by the comparison between open and hidden medical treatments (Levine, Gordon \& Fields, 1978; Levine \& Gordon, 1984; Amanzio, Pollo, Maggi \& Benedetti, 2001; Benedetti, Pollo, Lopiano, Lanotte, Vighetti \& Rainero, 2003). An open injection of a painkiller in full view of the patient, which represents usual medical practice, is more effective than a hidden injection. This hidden procedure completely eliminates the context associated with the knowledge that a pharmacological agent is being injected. The difference between the outcome of an open and hidden injection represents the placebo effect, at least its major component, which results from the patient's perception of the administration of the agent (Price, 2001).

Symbolic characteristics of the medication can influence nocebo effect. For example, historical reputation of some medications increases the likelihood to have adverse side effect ascribed to them. It is the case of penicillin. Up to $10 \%$ of hospitalized patients report penicillin allergy (Sørensen \& Kjaerulff, 1986), but some studies found that $94 \%$ of children (Graff-Lonnevig, Hedlin \& Lindfors, 1988) and 97\% of adults (Surtees, Stockton \& Gietzen, 1991) labeled as "penicillin allergic" tolerate oral penicillin. These patients may have mistakenly interpreted some coincidental symptoms as allergic in origin and the fear of a reaction deprive them of an effective treatment. Even the physical characteristics of the drugs influence the nature and the likelihood of side effect, like color, size, shape or name. For example, cold colors of the pills are associated with sedative effect, while warm ones suggest stimulant effect (de Craen, Roos, Leonard de Vries \& Kleijnen, 1996). More drowsiness is reported by volunteers taking blue placebo than those taking pink ones (Blackwell, Bloomfield \& Buncher, 1972). 


\section{Neurobiology of Negative eXPeCtations}

Latest scientific evidence indicates that these effects, stem from highly active processes in the Central Nervous System (CNS). During the anticipation of pain, several brain regions have been found to be activated (Chua, Krams, Toni, Passingham \& Dolan, 1999; Hsieh, Stone-Elander \& Ingvar, 1999; Ploghaus et al., 1999; Porro et al., 2002; Porro, Cettolo, Francescato \& Baraldi, 2003; Lorenz et al., 2005). For example, in a study led by Sawamoto et al. (2000), they used an innocuous thermal stimulation, founding that expectation of a painful stimulus amplified the perceived unpleasantness, and that these subjective hyperalgesic reports were accompanied by increased brain activations in the Anterior Cingulate Cortex (ACC), the Parietal Operculum (PO), and Posterior Insula (PI). Koyama, McHaffie, Laurienti, and Coghill (2005) noted that activation increased in the thalamus, insula, Pre-Frontal Cortex (PFC), and $\mathrm{ACC}$ as the magnitude of expected pain grew. By contrast, expectations of decreased pain reduced activation of pain-related brain regions, like the primary somatosensory cortex, the insular cortex, and ACC. Likewise, Keltner, Furst, Fan, Redfern, Inglis, and Fields (2006) found that the level of expected pain intensity altered the perceived intensity of pain along with the activation of different brain regions, like the ipsilateral caudal ACC, the head of the caudate, the cerebellum, and the contralateral nucleus cuneiformis (nCF).

Pharmacological studies are fundamental to understand the biochemistry of the nocebo effect and of negative expectations.

The major neurotransmitter implicated in the genesis of nocebo effects is the cholecystokinin (CCK). Benedetti, Amanzio, Casadio, Oliaro, and Maggi (1997) showed that nocebo hyperalgesia could be prevented by pretreatment with proglumide, a nonspecific cholecystokinin (CCK) antagonist for both CCK-A and CCK-B receptors, suggesting the possible involvement of CCK-ergic systems in the nocebo effect.

The involvement of CCK in nocebo hyperalgesia is likely to be mediated by anxiety, as benzodiazepines have been found to block both noceboinduced hyperalgesia and the typical anxiety-induced hypothalamus-pituitary-adrenal hyperactivity. Conversely, the CCK antagonist, proglumide, has been found to prevent nocebo hyperalgesia but not the hypothalamuspituitary-adrenal hyperactivity, which suggests two independent biochemical pathways activated by nocebo suggestions and anxiety (Benedetti, Amanzio, Vighetti \& Asteggiano, 2006).

In healthy volunteers, diazepam was able to block the nocebo response (Scott, Stohler, Egnatuk, Wang, Koeppe \& Zubieta, 2008). These curious responses are not entirely dependent on conscious perception of cues since they may be generated by unconscious cues as well (Jensen et al., 2012). 


\section{Clinical Research}

Compared with placebo research, little attention has been directed to clinical studies of nocebo effects and the frequency of the nocebo effects in clinical trials has not been as well documented. However, the nocebo effect is widely prevalent in clinical trials. Volunteers in the placebo-arm of trials often report adverse effects to the sham treatment. In studies of neurological disorders, the percentage of reported adverse effects in the placebo-arm has been as high as 64-74\% (Mitsikostas, 2012; Stathis, Smpiliris, Konitsiotis \& Mitsikostas, 2013). Furthermore, Mitsikostas, Mantonakis, and Chalarakis (2011) noted that the rates of the nocebo effect seen in clinical trials may be underestimated, as patients who are reluctant to receive novel medical treatments due to anxiety or mistrust, so more susceptible to nocebo response, might avoid participation in a clinical trial. In addition to this, a common nocebo effectrelated problem in clinical trials is the discontinuation and withdrawal by research subjects because of the severity of some side effects. In a pooled analysis of neuropathic pain trials, $6 \%$ of patients dropped out due to nonspecific nocebo- related adverse effects (Papadopoulos \& Mitsikostas, 2012).

Researchers distinguish between "apparent" and "true" placebo effects. The apparent placebo effect may be due to factors such as the natural history of the condition under study. On the contrary, a true placebo effect is attributed to the placebo intervention (Ernst \& Resch, 1995). A similar distinction cannot be extrapolated to nocebo effect. Apparent nocebo effects are adverse responses observed in the placebo-arm of a randomized controlled trial (Colloca \& Miller, 2011). Contrarily, true nocebo effect in double-blind drug trials includes all negative effects in placebo groups minus non-specific factors such as symptoms from the treated disease or co-morbid conditions, and adverse events of accompanying medication (Häuser, Hansen \& Enck, 2012).

Evaluating side effects of drugs is also a problem. The methods used for recording adverse effects changes the type and the frequency of effects reported: if a patients checks off a standardized list of symptoms, he specifies more adverse events with respect to reporting them spontaneously (Rief et al., 2009).

\section{Clinical practice}

Not only nocebo phenomena contribute to the outcomes of clinical trials (where it is going to be relatively straightforward to quantify their impact), but they also contribute to an unquantifiable extent, influence the outcomes 
of routine clinical care, affecting both patients' and clinicians' experiences. In everyday clinical practice, nocebo effects stem from interactions between physician and patient, patient and patient, and the general psychosocial context surrounding the patient and intervention. Physicians have an impact on patients and healing processes not only through drugs, interventions and therapy but also significantly by their words and personality. The verbal and non-verbal communications of the doctors and other staff do contain a lot of unintentional negative suggestions that may induce a nocebo response. Every symptom of an illness, side effect or complication can also be triggered by the wrong way of talking about it. Lang et al. (2000) observed that the patients receiving injections of radiographic medications showed heightened levels of anxiety and pain with the use of negative words such as "sting", "burn", "hurt", "bad", and "pain", when explaining the procedure. Moreover, patients perceive medical situations, such as an emergency, anesthesia or intensive care as extreme or even as life-threatening. This can induce a natural trance, an altered state of consciousness characterized by increased suggestibility (Cheek, 1962). Suggestions affect mental functions, such as anxiety and pain as well as physical functions. Strong figurative words, ambiguity, misunderstandings, incidental conversations, medical jargon and risk information can easily negatively impact the well-being of the patient (Zech, Seemann \& Hansen, 2014). Accordingly to Ashraf, Saaiq, and KhaleeqUz-Zaman (2014) the nocebo phenomenon is more frequently observed in association with female gender of the involved professionals, residency status versus faculty position and shorter professional experience (i.e. $<5$ years).

Physicians ought to be able to recognize if a side effect is more properly ascribed to the patient than to the treatment. A vague non-specific medication side effect is more likely due to nocebo response than drug-specific side effects (Davies, Jackson, Ramsay \& Ghahramani, 2003). If a non-serious symptom is idiosyncratic and not dose-dependent, it can be classified as nonspecific side effect (Barsky et al., 2002). These types of symptoms include difficulty concentrating, drowsiness, nausea, dizziness, fatigue, headache, insomnia, and overall poor health conditions. Conversely, specific side effects are physiological changes genuinely related to the pharmacological action and biological activity of the drug involved and tend to be dose-dependent.

There are a lot of management strategies to alleviate nocebo effect in clinical practice. Physicians should know the right amount of information to give to their patients. Obviously, they should communicate certain drugspecific life threatening adverse effects. Similarly, side effects with significant impact on quality of life should also be revealed but preferably with positive framing. Informing the patient about every possible minor adverse effect of the drug is clearly unwarranted. 
The probability of experiencing adverse effects can be communicated qualitatively and/or quantitatively (or statistically). Qualitative information may be expressed "negatively" (by focusing on the minority of patients who experience a particular side effect) or "positively" (by focusing on most patients who do not experience the side effect) (Colloca \& Miller, 2011). A positive framing should minimize nocebo responses, as well as reassuring on reversibility of adverse effects on cessation of the drug (wherever applicable).

Quantitative or statistical information from published scientific data may help to downplay nocebo response. The combination of quantitative information with positive framing gives a positive effect. This was demonstrated in a study evaluating adverse effects and work absenteeism after influenza immunization. Patients who were apprised about the percentage of patients free of vaccine side effects (positive framing) reportedly had significantly lesser adverse effects than patients who were informed about their occurrence (negative framing) (O'Connor, Pennie \& Dales, 1996).

Physicians and other medical staff should discourage the patients on "non-professional" opinion seeking behavior. Patients should be cautioned against forming their opinion based on information taken from other patients and internet portals that lacks scientific evidence. In addition to this, if side effects occur, physicians should find out if the patient has any dissatisfaction with his medical care. Though it may be not possible to accommodate the patient's concerns, elucidating and discussing them may help to reestablish a collaborative alliance (Barsky et al., 2002). Patient counseling with the goal of minimizing nocebo effect is a continuous process requiring reinforcements during subsequent patient- physician encounters.

In order to minimize nocebo effects consistent with patient autonomy, a technique of "authorized concealment" is worth discussing. In this approach, patients are asked if they are willing to agree not to receive information about certain types of side effects of the prescribed drug (Colloca \& Miller, 2011). Authorized concealment may be appropriate for relatively mild and/or transient side effects while serious or irreversibly harmful side effect should not be concealed.

Another way to taking into account nocebo phenomenon is the "contextualized informed consent". One of the primary aims of physicians is the principle of XXX non-maleficence. At the same time, the major point of modern bioethics is informed consent, respect for person, and transparency (Gillon, 2003). One can abide by both principles through "contextualized informed consent". This involves taking into account the possible side effects, the person being treated, and the disease involved in order to adapt the information provided about medication side effects to provide the most transparency with the least potential harm (Wells \& Kaptchuck, 2012). 


\section{ETHICAL IMPLICATIONS}

As stewards of knowledge, the physician are therapeutic agents who must employ information to make technically "right" decisions, to give treatment based upon a mechanistic understanding of the pathologic process. However, what is technically "right" may not be biomedically or ethically "good" for a particular patient along the disease -illness continuum. Hence, the physicians should be morally capable to render an ethically "good" treatment (Pellegrino, 1979; 1983). Certainly, one form of knowledge is scientific; contextually this involves identifying the neurological mechanisms and processes that subserve pain in all of its dimensions (i.e., sensory, perceptual, cognitive, etc.), and how these neural substrates can produce placebo and/or nocebo effects and responses. This knowledge derives from research, and represents the actual basis of applied biomedicine. However, such a "secular" understanding of neural function may be insufficient to fully appreciate the dilemma of pain for a particular patient and allow reasoning necessary to render a treatment that is biomedically and ethically good for that patient. Indeed, medicine is more than simply applied biology. Knowledge must be gained through diverse technical and subjective experiences acquired over time that affect first-hand interactions with individual patients (Giordano \& Boswell, 2005).

\section{CONClusions AND FUtURE PERSPECTIVES}

From the point of view of a patient, non specific side effects could be very distressing. They could result in wasted medication and nonhaderence, unnecessary physician visits and complicated regimens deriving from the addition of drugs to treat the side effect. As well as being distressing, this phenomenon could also become very expensive. Moreover, accordingly to Ashraf et al. (2014), the current knowledge of medical staff about the nocebo phenomenon is less than ideal.

For all these reasons, nocebo phenomenon deserves greater clinical attention and more empirical studies to assess techniques of clinician-patient interaction that can minimize nocebo effect consistent with informed consent and respect for patient autonomy. 


\section{REFERENCES}

Amanzio, M., Pollo, A., Maggi, G., \& Benedetti, F. (2001). Response variability to analgesics: a role for non-specific activation of endogenous opioids. Pain, 90 (3), 205-215.

Ashraf, B., Saaiq, M., \& Uz-Zaman, K. (2014). Qualitative study of Nocebo Phenomenon (NP) involved in doctor-patient communication. International Journal of Health Policy and Management, 3, 23-27.

Barsky, A.J. (1983). Nonpharmacologic aspects of medication. Archives of Internal Medicine, 143 (8), 1544-1548.

Barsky, A.J., Saintfort, R., Rogers, M.P., \& Borus, J.F. (2002). Nonspecific medication side effects and the nocebo phenomenon. Jama, 287 (5), 622-627.

Benedetti, F., Amanzio, M., Casadio, C., Oliaro, A., \& Maggi, G. (1997). Blockade of nocebo hyperalgesia by the cholecystokinin antagonist proglumide. Pain, 71 (2), 135-140.

Benedetti, F., Amanzio, M., Vighetti, S., \& Asteggiano, G. (2006). The biochemical and neuroendocrine bases of the hyperalgesic nocebo effect. The Journal of Neuroscience, 26 (46), 12014-12022.

Benedetti, F., Pollo, A., Lopiano, L., Lanotte, M., Vighetti, S., \& Rainero, I. (2003). Conscious expectation and unconscious conditioning in analgesic, motor, and hormonal placebo/nocebo responses. The Journal of Neuroscience, 23 (10), 4315-4323.

Bingel, U., Wanigasekera, V., Wiech, K., Mhuircheartaigh, R.N., Lee, M.C., Ploner, M., \& Tracey, I. (2011). The effect of treatment expectation on drug efficacy: imaging the analgesic benefit of the opioid remifentanil. Science Translational Medicine, 3 (70), 70ra14.

Blackwell, B., Bloomfield, S., \& Buncher, C.R. (1972). Demonstration to medical students of placebo responses and non-drug factors. The Lancet, 299 (7763), 1279-1282.

Cheek, D.B. (1962). Importance of recognizing that surgical patients behave as though hypnotized. American Journal of Clinical Hypnosis, 4 (4), 227-236.

Chua, P., Krams, M., Toni, I., Passingham, R., \& Dolan, R. (1999). A functional anatomy of anticipatory anxiety. Neuroimage, 9 (6), 563-571.

Colloca, L., \& Miller, F.G. (2011). The nocebo effect and its relevance for clinical practice. Psychosomatic Medicine, 73 (7), 598-603.

Davies, S.J., Jackson, P.R., Ramsay, L.E., \& Ghahramani, P. (2003). Drug intolerance due to nonspecific adverse effects related to psychiatric morbidity in hypertensive patients. Archives of Internal Medicine, 163 (5), 592-600.

De Craen, A.J., Roos, P.J., De Vries, A.L., \& Kleijnen, J. (1996). Effect of colour of drugs: systematic review of perceived effect of drugs and of their effectiveness. BMJ: British Medical Journal, 313 (7072), 1624-1626. 
Ernst, E., \& Resch, K.L. (1995). Concept of true and perceived placebo effects. BMJ: British Medical Journal, 311 (7004), 551.

Flaten, M.A., Simonsen, T., \& Olsen, H. (1999). Drug-related information generates placebo and nocebo responses that modify the drug response. Psychosomatic Medicine, 61 (2), 250-255.

Gillon, R. (2003). Ethics needs principles - four can encompass the rest - and respect for autonomy should be "first among equals". Journal of Medical Ethics, 29 (5), 307-312.

Giordano, J., \& Boswell, M.V. (2005). Pain, placebo, and nocebo: epistemic, ethical, and practical issues. Pain Physician, 8 (4), 331-333.

Graff-Lonnevig, V., Hedlin, G., \& Lindfors, A. (1988). Penicillin allergy: a rare paediatric condition? Archives of Disease in Childhood, 63 (11), 1342-1346.

Häuser, W., Hansen, E., \& Enck, P. (2012). Nocebo phenomena in medicine: their relevance in everyday clinical practice. Deutsches Ärzteblatt International, 109 (26), 459-465.

Hsieh, J.C., Stone-Elander, S., \& Ingvar, M. (1999). Anticipatory coping of pain expressed in the human anterior cingulate cortex: a positron emission tomography study. Neuroscience Letters, 262 (1), 61-64.

Keltner, J.R., Furst, A., Fan, C., Redfern, R., Inglis, B., \& Fields, H.L. (2006). Isolating the modulatory effect of expectation on pain transmission: a functional magnetic resonance imaging study. The Journal of Neuroscience, 26 (16), 4437-4443.

Kennedy, W.P. (1961). The nocebo reaction. Medical World, 95, 203-205.

Koyama, T., McHaffie, J.G., Laurienti, P.J., \& Coghill, R.C. (2005). The subjective experience of pain: where expectations become reality. Proceedings of the National Academy of Sciences of the United States of America, 102 (36), 1295012955.

Jensen, K.B., Kaptchuk, T.J., Kirsch, I., Raicek, J., Lindstrom, K.M., Berna, C., Gollup, R.L., Ingvar, M., \& Kong, J. (2012). Nonconscious activation of placebo and nocebo pain responses. Proceedings of the National Academy of Sciences, 109 (39), 15959-15964.

Lang, E.V., Benotsch, E.G., Fick, L.J., Lutgendorf, S., Berbaum, M.L., Berbaum, K.S., Logan, H., \& Spiegel, D. (2000). Adjunctive non-pharmacological analgesia for invasive medical procedures: a randomised trial. The Lancet, 355 (9214), 1486-1490.

Lees-Haley, P.R., \& Brown, R.S. (1992). Biases in perception and reporting following a perceived toxic exposure. Perceptual and Motor Skills, 75 (2), 531-544.

Levine, J.D., \& Gordon, N.C. (1984). Influence of the method of drug administration on analgesic response. Nature, 312 (5996), 755-756.

Levine, J., Gordon, N., \& Fields, H. (1978). The mechanism of placebo analgesia. The Lancet, 312 (8091), 654-657. 
Lorenz, J., Hauck, M., Paur, R.C., Nakamura, Y., Zimmermann, R., Bromm, B., \& Engel, A.K. (2005). Cortical correlates of false expectations during pain intensity judgments: a possible manifestation of placebo/nocebo cognitions. Brain, Behavior, and Immunity, 19 (4), 283-295.

Mitsikostas, D.D., Mantonakis, L.I., \& Chalarakis, N.G. (2011). Nocebo is the enemy, not placebo: a meta-analysis of reported side effects after placebo treatment in headaches. Cephalalgia, 31 (5), 550-561.

Mitsikostas, D.D. (2012). Nocebo in headaches: implications for clinical practice and trial design. Current Neurology and Neuroscience Reports, 12 (2), 132-137.

Moerman, D.E. (1981). Edible symbols: the effectiveness of placebos. Annals of the New York Academy of Sciences, 364 (1), 256-268.

Morrow, G.R., Roscoe, J.A., Kirshner, J.J., Hynes, H.E., \& Rosenbluth, R.J. (1998). Anticipatory nausea and vomiting in the era of 5-HT3 antiemetics. Supportive Care in Cancer, 6 (3), 244-247.

Myers, M.G., \& Cairns, J.A. (1987). The consent form as of side effects. Clinical, Pharmacology and Therapeutics, 42 (3), 250-253.

O'Connor, A.M., Pennie, R.A., \& Dales, R.E. (1996). Framing effects on expectations, decisions, and side effects experienced: the case of influenza immunization. Journal of Clinical Epidemiology, 49 (11), 1271-1276.

Papadopoulos, D., \& Mitsikostas, D.D. (2012). A meta-analytic approach to estimating nocebo effects in neuropathic pain trials. Journal of Neurology, 259 (3), 436-447.

Pellegrino, E.D. (1979). The anatomy of clinical judgments: some notes on right reason and right action. In: Engelhardt, H.T., Spicker, S.F., \& Towers, B. (eds.), Clinical judgment: a critical appraisal. The Netherlands: Springer, pp. 169-194.

Pellegrino, E.D. (1983). The healing relationship: the architectonics of clinical medicine. In: Shelp, E. (ed.), The clinical encounter: the moral fabric of the patient-physician relationship. Netherlands: Springer, pp. 153-172.

Ploghaus, A., Tracey, I., Gati, J.S., Clare, S., Menon, R.S., Matthews, P.M., \& Rawlins, J.N.P. (1999). Dissociating pain from its anticipation in the human brain. Science, 284 (5422), 1979-1981.

Porro, C.A., Baraldi, P., Pagnoni, G., Serafini, M., Facchin, P., Maieron, M., \& Nichelli, P. (2002). Does anticipation of pain affect cortical nociceptive systems? The Journal of Neuroscience, 22 (8), 3206-3214.

Porro, C.A., Cettolo, V., Francescato, M.P., \& Baraldi, P. (2003). Functional activity mapping of the mesial hemispheric wall during anticipation of pain. Neuroimage, 19 (4), 1738-1747.

Price, D.D. (2001). Assessing placebo effects without placebo groups: an untapped possibility? Pain, 90 (3), 201-203. 
Rief, W., Nestoriuc, Y., von Lilienfeld-Toal, A., Dogan, I., Schreiber, F., Hofmann, S.G., Barsky, A.J., \& Avorn, J. (2009). Differences in adverse effect reporting in placebo groups in SSRI and tricyclic antidepressant trials: a systematic review and meta-analysis. Drug Safety, 32 (11), 1041-1056.

Sawamoto, N., Honda, M., Okada, T., Hanakawa, T., Kanda, M., Fukuyama, H., Konishi, J., \& Shibasaki, H. (2000). Expectation of pain enhances responses to nonpainful somatosensory stimulation in the anterior cingulate cortex and parietal operculum/posterior insula: an event-related functional magnetic resonance imaging study. The Journal of Neuroscience, 20 (19), 7438-7445.

Schweiger, A., \& Parducci, A. (1981). Nocebo: the psychologic induction of pain. The Pavlovian Journal of Biological Science: Official Journal of the Pavlovian, 16 (3), 140-143.

Scott, D.J., Stohler, C.S., Egnatuk, C.M., Wang, H., Koeppe, R.A., \& Zubieta, J.K. (2008). Placebo and nocebo effects are defined by opposite opioid and dopaminergic responses. Archives of General Psychiatry, 65 (2), 220-231.

Sørensen, H.T., \& Kjaerulff, E. (1986). Is type I penicillin allergy overdiagnosed in children? Scandinavian Journal of Primary Health Care, 4 (4), 252-253.

Stathis, P., Smpiliris, M., Konitsiotis, S., \& Mitsikostas, D.D. (2013). Nocebo as a potential confounding factor in clinical trials for Parkinson's disease treatment: a meta-analysis. European Journal of Neurology, 20 (3), 527-533.

Surtees, S.J., Stockton, M.G., \& Gietzen, T.W. (1991). Allergy to penicillin: fable or fact? BMJ: British Medical Journal, 302 (6784), 1051-1052.

Wells, R.E., \& Kaptchuk, T.J. (2012). To tell the truth, the whole truth, may do patients harm: the problem of the nocebo effect for informed consent. The American Journal of Bioethics, 12 (3), 22-29.

Zech, N., Seemann, M., \& Hansen, E. (2014). Nocebo effects and negative suggestion in anesthesia. Anaesthesist, 63 (11), 816-824. 